\title{
Measuring movement fluency during the sit-to-walk task
}

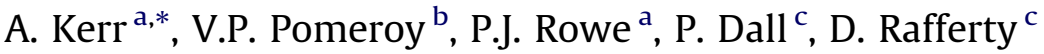 \\ a Department of Biomedical Engineering, University of Strathclyde, 106 Rottenrow, Glasgow G4 0NW, United Kingdom \\ ${ }^{\mathrm{b}}$ Faculty of Medicine and Health Sciences, University of East Anglia, Queen's Building, Norwich NR4 7TJ, United Kingdom \\ ' School of Health and Life Sciences, Glasgow Caledonian University, Cowcaddens Road, Glasgow G4 OBA, United Kingdom
}

\section{A R T I C L E I N F O}

\section{Article history:}

Received 9 December 2011

Received in revised form 19 September 2012

Accepted 30 September 2012

\section{Keywords:}

Fluency

Smoothness

Co-ordination

Sit-to-walk

\begin{abstract}
A B S T R A C T
Background: Restoring movement fluency is a key focus for physical rehabilitation; it's measurement, however, lacks objectivity. The purpose of this study was to find whether measurable movement fluency variables differed between groups of adults with different movement abilities whilst performing the sitto-walk (STW) movement. The movement fluency variables were: (1) hesitation during movement (reduction in forward velocity of the centre of mass; CoM), (2) coordination (percentage of temporal overlap of joint rotations) and (3) smoothness (number of inflections in the CoM jerk signal).

Methods: Kinematic data previously collected for another study were extracted for three groups: older adults $(n=18)$, older adults at risk of falling (OARF, $n=18$ ), and younger adults $(n=20)$. Each subject performed the STW movement freely while a motion analysis system tracked 11 body segments. The fluency variables were derived from the processed kinematic data and tested for group variation using analysis of variance.

Findings: All three variables showed statistically significant differences among the groups. Hesitation $(F=15.11, p<0.001)$ was greatest in the OARF 47.5\% (SD 18.0), compared to older adults 30.3\% (SD 15.9) and younger adults $20.8 \%$ (SD 11.4). Co-ordination $(F=44.88, p<0.001)$ was lowest for the OARF (6.93\%, SD 10.99) compared to both the young (31.21\%, SD 5.48) and old (26.24\%, SD 5.84). Smoothness $(F=35.96, p<0.001$ ) was best in the younger adults, 18.3 (SD 5.2) inflections, compared to the old, 42.5 (SD 11.5) and OARF, 44.25 (SD 7.29).

Interpretation: Hesitation, co-ordination and smoothness may be valid indicators of movement fluency in adults, with important consequences for research and clinical practice.
\end{abstract}

(c) 2012 Elsevier B.V. All rights reserved.

\section{Introduction}

Visual observation of movement fluency is used by therapists to guide the planning and evaluation of interventions aimed at restoring movement [27]. Improving movement fluency is perceived by therapists to be associated with improvements in function [6], and has been shown to relate to energy cost [33]. It is a key objective of the predominant approach to neurorehabilitation in the United Kingdom [23,31]. There is an underlying assumption, however, that therapists have a tacit and common understanding of what constitutes fluent movement [28]. This assumption, rarely tested, could undermine the rigour of clinical assessment and create an obstacle to the scientific scrutiny of physical rehabilitation.

Tools for measuring movement fluency have been developed previously using normal movement as a reference for trained observers [2]. In some cases these scales have demonstrated good

\footnotetext{
* Corresponding author.

E-mail address: a.kerr@strath.ac.uk (A. Kerr).
}

prognostic and diagnostic properties [26]. However their subjective nature causes reliability problems [21], and they rely on substantial experience and training of observers [20]. To avoid these hindrances Malouin et al. [19] introduced an objective method for quantifying fluency based on a threshold drop in forward momentum while performing the sit-to-walk (STW) movement. The resulting dichotomous (fluent or not fluent) index was subsequently adapted into a clinical scale by Dion et al. [8]. This objective method of judging movement fluency from a change in forward momentum is promising; with evidence of validity as a screening tool for mobility problems, e.g. identifying a risk of falling (sensitivity 0.96 and specificity 0.89 ) [15]. However, as a measure with a single dimension, it lacks content validity.

To improve patient assessment and provide a research tool for the study of movement disorders and their rehabilitation, valid, objective measurements of movement fluency are needed [20]. The multifarious nature of movement fluency, and lack of clear definition, may contribute to the paucity of objective measures. A way forward is to deconstruct fluency into separate variables that can be clearly defined, are clinically useful, and, critically, can be measured. 
Normal movement is characteristically smooth, co-ordinated and efficient [30] without unnecessary hesitation or repetition. A working definition of fluent movement, therefore, should include measurable definitions of these terms: hesitation, co-ordination and smoothness.

Movement hesitation is frequently described in neurological conditions [9,22] and musculoskeletal conditions [13] but less frequently quantified. The freezing of gait questionnaire [12] has been used in Parkinson's disease to rate frequency and magnitude of movement hesitations with good reliability (Cronbach alpha $=0.94$ ) and validity (correlation to a standard function scale during the "on" period was 0.66) but is a subjective, disease specific, tool. Using motion analysis technology both Kerr et al. [15] and Malouin et al. [19] quantified movement hesitation during the STW movement as a percentage drop in forward movement of the centre of mass, and this is probably the only reported objective method for measuring hesitation during movement.

Co-ordination is consistently reported as a characteristic of normal fluent movement, for upper and lower limb movements, although the term tends to be applied generically, without reference to a measurable definition [23]. Metrics based around the relative timing of joint movements, most notably temporal overlapping, have been developed [16,17] as possible objective measures of co-ordination with higher percentages of temporal overlap indicating a more fluent movement.

Movement smoothness, and associated terms such as quality and efficiency of movement are used by therapists to express impaired motor control as well as a guiding principle for therapy $[5,23,31]$. The absence of a measurable definition, however, hampers both scientific scrutiny and patient assessment. Definitions based on jerk (third time derivative of position) have been applied to upper limb therapeutic robots with mean and variation calculated as metrics of smoothness [25].

Although this method could be applied to any movement, as yet it has not been applied to whole body movements such as walking or STW.

The merging of two separate movements during the STW movement (sit-to-stand and gait initiation [18]) has been recognised as a suitable testing ground for movement fluency $[1,3,8,11,15]$. Sit-to-walk is a functional, whole body movement. To date, however, the only fluency measurement recorded for the STW movement has been the drop in CoM velocity, previously mentioned.

The primary aim of this paper was to present novel analytical tools to express movement fluency in a manner that is sympathetic to therapy objectives and which may uncover new understanding of human movement. The secondary aim was to determine if any of these tools could statistically distinguish groups of individuals with expected differences in movement fluency when performing the STW movement.

\section{Methods}

\subsection{Design and ethics}

Biomechanical data were extracted from the electronic records of a previous observational study of the STW movement [15], which had ethical approval from the University and NHS ethics committees. All participants gave written informed consent for the earlier study.

\subsection{Participants}

Three groups were recruited: (1) young adults $(n=20$, age 33.1 years (SD, 8), mass $71.9 \mathrm{~kg}$ (SD, 11.6), height $1.72 \mathrm{~m}$ (SD, 0.1$)$ ), (2) older adults $(n=18$, age 70.3 years (SD, 5.4), mass $65.8 \mathrm{~kg}(\mathrm{SD}, 25.8)$, height $1.66 \mathrm{~m}(\mathrm{SD}, 0.1)$ ) and (3) older adults at risk of falling (OARF) $(n=18$, age 79.6 years (SD, 7.5), mass $66.2 \mathrm{~kg}$ (SD, 16.7), height $1.53 \mathrm{~m}$ (SD 0.1)). Participants were recruited from the local community (old and young groups) and a falls prevention programme (OARF group), respectively.

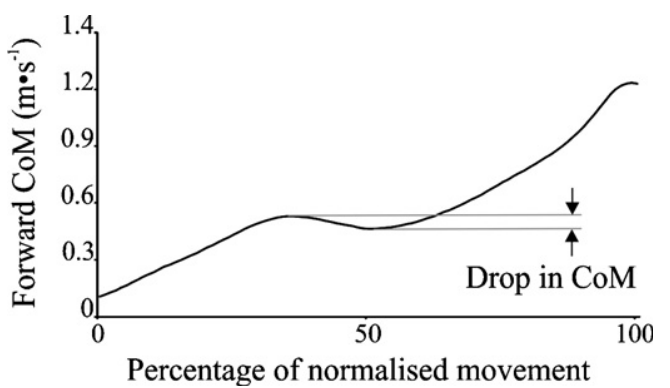

Fig. 1. Hesitation; defined as the percentage drop in forward velocity.

\subsection{Procedure}

All measurements were made in a movement laboratory. Participants wore tight fitting shorts, t-shirt and trainers and sat on a standard chair $(0.44 \mathrm{~m}$ in height, armless but with a $10^{\circ}$ reclined backrest). Reflective markers (diameter $0.02 \mathrm{~m}$, mass $0.03 \mathrm{~kg}$ ) were mounted onto the skin and, where necessary, the clothing overlying anatomical landmarks so that 11 segments (feet, lower legs, thighs, trunk, upper and lower arms) could be constructed using a rigid body model [24].

\subsection{The movement task}

Each participant started the test in sitting, and was requested to walk to another chair placed six metres in front of them. Participants selected their starting position and performed the movement in their own preferred manner. Each participant performed five repetitions of the movement with data from the first three successful (all marker trajectories captured) repetitions used for analysis. Participants were given up to $5 \mathrm{~min}$ to rest between repetitions.

\subsection{Equipment}

\subsubsection{Marker}

Marker trajectories were tracked in three dimensions by seven Motion Capture Units (Qualisys Medical AB, Gothenburg, Sweden). Data were recorded at a sampling rate of $50 \mathrm{~Hz}$ and the system calibrated to collect a volume of $4 \mathrm{~m}$ (saggital plane $-X$ axis - direction of travel) by $1.8 \mathrm{~m}$ ( $Z$ axis - height) by $1.5 \mathrm{~m}$ ( $Y$ axis coronal plane) metres using Qualysis TrackManager.

\subsection{Data analysis}

Marker trajectories were filtered using a low-pass 4th order Butterworth filter with cut-off frequency of $6 \mathrm{~Hz}$, and interpolated with a maximum gap fill of 10 frames using a non-uniform rational B-spline [10]. The resulting data were used to construct a model of the body using Visual 3D (Version 3.28, C-Motion, Inc., Rockville, MD, USA) allowing calculation of joint angles and total body centre of mass (CoM) in three dimensions.

The onset time (first continuous forward movement of the CoM) and end time (end of the first swing phase, i.e. first initial contact) were recorded for each movement to allow normalisation of the data to $100 \%$ of the movement. The three fluency variables: hesitation, co-ordination and smoothness, were then calculated from the resulting processed data.

Hesitation was defined as the maximum drop in forward velocity of the COM from the initial peak generated during the seated phase, expressed as a percentage of the initial peak value, see Fig. 1.

Co-ordination was calculated as the temporal overlap between the knee and hip in the saggital plane (as a percentage of the whole movement time) during two

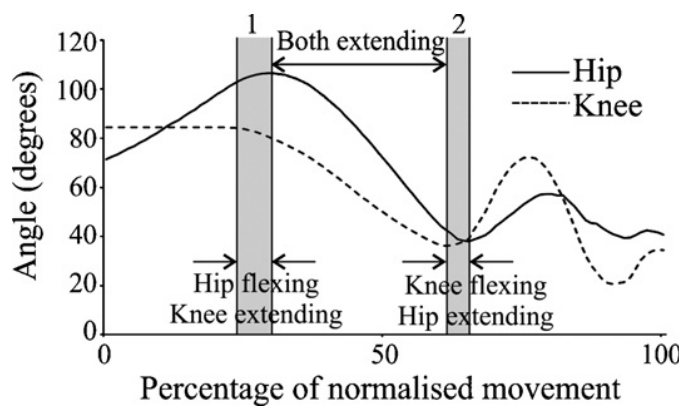

Fig. 2. Co-ordination; defined as the temporal overlap between hip and knee movements in the saggital plane. 


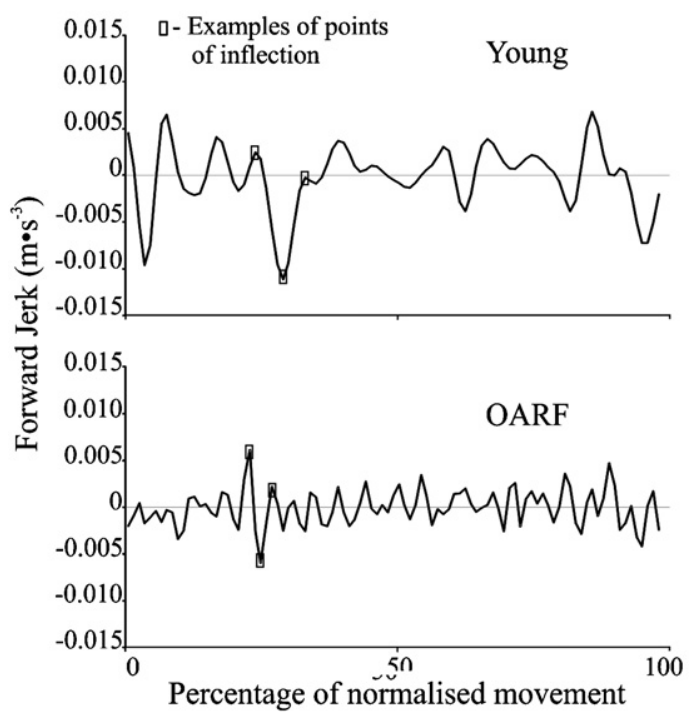

Fig. 3. Smoothness; defined as the count of inflections in the jerk signal, data from two different participants.

periods (1) end of initial hip flexion and start of knee extension (time of knee extension - time of hip flexion ending) and (2) end of hip extension while rising and the start of knee flexion (time of knee flexion - time of hip extension ending) see Fig. 2.

Smoothness was calculated as the number of inflections in the CoM jerk signal, an inflection was identified by a logic statement (Microsoft Excel 2007) that determined a negative change in the signal (a time point greater than the previous two time points and greater than the two subsequent time points) or positive change (a time point less the previous two time points and less than the two subsequent time points) as illustrated in Fig. 3.

Each of the fluency variables was compared among the groups using a one factor ANOVA. Significant effects $(p<0.05)$ were then explored with a Tukey test. All statistical analyses were performed in Minitab (version 16).

\section{Results}

\subsection{Hesitation}

There was a drop in CoM forward velocity in all groups but with significant differences in magnitude $(F=15.11, p<0.001)$. The OARF group showed the biggest drop (47.51\%, SD 18.00) followed by the old group (30.29\%, SD 15.86) and then the young group (20.82\%, SD 11.43), see Table 1 . Post hoc analysis confirmed the statistical difference lay between the OARF and both young and old groups.

\subsection{Co-ordination}

All three groups had negative values at both overlap periods indicating:
(1) The knee begins to extend before the hip ends its flexion movement.

(2) The knee starts to flex (to start the first swing phase) before the hip ends the extension movement.

The magnitude of the overlap, however, varied among the groups during the first $(F=5.28, p=0.008)$ and second $(F=44.88$, $p<0.001)$ periods. Post hoc analysis identified the OARF (1st overlap $-1.47 \%$, SD 9.89 and 2nd overlap -6.93\%, SD 10.99) as being different to both young (1st overlap $-13.16 \%$, SD 9.63 and 2nd $-31.21 \%$, SD 5.48) and the old group (1st overlap -11.33 , SD 13.02 and 2 nd $-26.24 \% \pm 5.84$ ) groups at this second overlap point, see Table 1

\subsection{Smoothness}

Smoothness differed significantly among the groups $(F=35.96$, $p<.001)$, post hoc analysis identified the young group (18.25 inflections, SD 5.20) as different to both the old (42.50, SD 11.45) and OARF (44.25, SD 7.29), see Table 1.

\section{Discussion}

This study found significant differences in movement fluency among groups of younger adults, older adults and OARF performing the STW movement. As measures of fluency; hesitation, coordination and smoothness, were all found to vary statistically. The ability of these measures to discriminate groups of people expected to exhibit different characteristics of movement fluency raises hypotheses about the biomechanics of movement control.

Quantifying hesitation as a reduction in the body's velocity fits the meaning of fluidity. Fluid movement, after all, is continuous movement; ambiguity however exists as to what point a reduction in velocity means a loss of fluidity. While Dion et al. [8] selected a reduction of $30 \%$ or more in CoM forward velocity to indicate a non-fluid movement our data points to a value of $50 \%$. The occurrence of hesitation in all three groups suggests it is an invariant part of the movement. During this period (transition between sitting and standing) the body changes direction of movement from predominantly forward to predominantly upward, primarily through hip and knee extension. It appears that the young and old group were able to retain most of their initial forward momentum, suggesting greater mechanical efficiency, whereas the OARF reduced their forward momentum by $50 \%$. Allowing horizontal momentum to reduce by so much, at the cost of additional muscle work to regenerate the forward momentum for gait, is likely to be a deliberate strategy. A fear of falling, for example, could explain the need to minimise potentially destabilising momenta once standing. On the other hand, reducing momentum could simply reflect a greater, age related, delay in switching from one muscle synergy to another, in this case

Table 1

Descriptive and inferential statistics for the three fluency variables.

\begin{tabular}{|c|c|c|c|c|}
\hline Fluency variable & Young & Old & Old at risk falling & Anova \\
\hline Hesitation $^{\mathrm{a}}$ & $20.82(11.43)$ & $30.29(15.86)$ & $47.51(18.00)^{*}$ & $F=15.11, p<0.001$ \\
\hline Co-ordination $1^{\mathrm{b}}$ & $-13.16(9.63)$ & $-11.33(13.02)$ & $-1.47(9.89)^{*}$ & $F=5.28, p=0.008$ \\
\hline Co-ordination 2 & $-31.21(5.48)$ & $-26.24(5.84)$ & $-6.93(10.99)^{*}$ & $F=44.88, p<0.001$ \\
\hline Smoothness ${ }^{c}$ & $18.25(5.21)^{*}$ & $42.50(11.45)$ & 44.25 (7.29) & $F=35.96, p<0.001$ \\
\hline
\end{tabular}

a Hesitation: maximum percentage drop in forward velocity of the centre of mass (COM) from the initial peak generated during the seated phase.

b Co-ordination: temporal overlap (percent) between knee and hip movement in the saggital plane during two periods (1) end of initial hip flexion and start of knee extension, and (2) end of hip extension and the start of knee flexion.

c Smoothness: number of inflections (change in direction over 2 percentage points) in the CoM jerk signal.

Statistical difference according to post hoc analysis, Tukey. 
between flexion and extension of the joints. These hypotheses should be explored in future research.

Interruptions, or hesitations, in movement are common and are already included in some mobility tests due to their usefulness in screening for mobility problems, for example the stops walking when talking test [7]. Our quantification of hesitation with ratio, as opposed to categorical (e.g. did or did not stop walking), data might resolve some of the sensitivity issues with these tests [14].

Whereas hesitation represents a total body performance measure, the co-ordination calculation examined movement fluidity on an elemental scale (joint movement). This may be more helpful to therapists directly involved in rehabilitation. Compared to the OARF group, the two healthy groups (young and old) were similar in terms of the mean and variability of their coordination suggesting a similar movement strategy was adopted [11]. The first overlap period (around seat-off) saw the young and old groups overlapping their hip flexion and knee extension by $13 \%$ and $11 \%$, respectively, of the total time. Continued hip flexion while the knee starts to extend suggests an ability to maintain forward momentum while the body rises. The OARF group, however, had a very small overlap during this period $(<2 \%)$, so that hip flexion ended (reversing to extension) around the same time as the knee began to extend. The lack of overlap may be for mechanical reasons; coupling the extension movements of the knee and hip to maximise body lift.

The second period of overlap showed a similar pattern but with greater differences for the OARF group. The young and old groups were able to initiate gait (start of knee flexion) while continuing to rise (hip extension), this overlap was $31 \%$ and $26 \%$, respectively, and clearly shows an ability to merge two movement patterns, i.e. hip extension (rising from the chair) and knee flexion (initiating gait). This overlap was much smaller for the OARF group, although knee flexion did occur before hip extension ended, there was only a $7 \%$ overlap.

The difference, in magnitude of co-ordination, between the OARF and both healthy groups suggests different strategies were employed. The two healthy groups allowed extension and flexion of the lower limbs to occur simultaneously, this has several advantages: it reduces the movement time, maintains existing momentum, and takes advantage of stored elastic energy in the connective tissue [32]. This strategy is likely to be more efficient than that adopted by the OARF group. This group favoured a less fluid approach to the movement, enhancing stability by minimising horizontal momentum while rising and ensuring the body was upright and balanced before walking.

Our calculation of smoothness, based on jerk of the total body CoM, clearly differentiated the young group from both older groups (old and OARF). Considering the previous similarity between the young and old group for hesitation and co-ordination this difference was both surprising and intriguing. Smooth movement is not, necessarily, a characteristic of functional movement but it suggests a level of motor control has been attained, particularly when exhibited during a whole body movement that is inherently unstable, and requires distinct phases of acceleration, as in the case of STW [18]. The difference in smoothness uncovered between the young and old may represent fine scale changes in motor control, caused by age related changes in the neuromusculoskeletal system. As such our quantification of movement smoothness may be valuable to future researchers.

Calculations of movement smoothness have existed in robotic literature for some time, particularly in studies investigating the use of therapeutic robots [4] where the smoothness of the movement (typically upper limb) is used as an outcome measure. The method used in this paper was applied to the CoM movement which is a derived value from the movement of all tracked segments of the body (11 segments) and is therefore sensitive to positional change in any segment, e.g. rapid arm movement, to gain balance, will cause a jerk. Calculating the number of jerks in the movement was felt to be appropriate for a whole body movement, but it is also sensitive to changes that may not actually be important to the movement itself, for example placing the hands in a different position.

The discriminant validity of these measures could be useful in clinical practice. Considering the prominence of movement fluency in rehabilitation literature $[16,21,23,29]$, both diagnostically and as a guide to therapists, its measurement has potential value. The methods presented in this paper may not readily translate to clinical practice due to the instrumentation and data processing involved, however the strategic use of accelerometers and gyroscopes may provide a method of clinically applying these techniques. In terms of elegance of data, there is an argument that these three measures of movement fluency could be assimilated into one value, however, as the variables are all directly related to each other, a composite value would require further testing.

\section{Conclusion}

This paper outlined a method for measuring movement fluency during the STW movement. Applying them to three groups of people who were expected to exhibit different levels of movement control identified that all movement fluency variables had discriminant validity. The operational definitions and reference data can provide a framework for further development of both laboratory and clinically based measures to movement fluency.

\section{Funding}

There was no funding associated with this study or writing of this paper.

\section{Acknowledgements}

The authors would like to acknowledge the initial contribution of the late Dr. Kate Kerr and Professor Brian Durward. The time for Dr. Andrew Kerr was funded by the Efficacy and Mechanism Evaluation programme, funded by the MRC and NIHR, with contributions from the CSO in Scotland, NISCHR in Wales and the HSC R\&D, Public Health Agency in Northern Ireland.

\section{Conflict of interest statement}

The authors declare that there is no conflict of interest in the conduction of this study or writing of this paper.

\section{References}

[1] Aberg AC, Frykberg GE, Halvorsen K. Medio-lateral stability of sit-to-walk performance in older individuals with and without fear of falling. Gait and Posture 2010;31(4):438-43.

[2] Boyce WF, Gowland C, Rosenbaum PL, Lane M, Plews N, Goldsmith C, et al Measuring quality of movement in cerebral palsy: a review of instruments. Physical Therapy 1991;71(11):813-9.

[3] Buckley TA, Pitsikoulis C, Hass CJ. Dynamic postural stability during sit-towalk transitions in Parkinson disease patients. Movement Disorders Official Journal of The Movement Disorder Society 2008;23(9):1274-80.

[4] Celik O, Malley MKO, Boake C, Levin HS, Yozbatiran N, Reistetter TA. Normalized movement quality measures for therapeutic robots strongly correlate with clinical motor impairment measures. IEEE Transactions on Neural and Rehabilitation Systems Engineering 2010;18(4):11.

[5] Cirstea MC, Levin MF. Compensatory strategies for reaching in stroke. Brain 2000;123(5):940-53.

[6] Davidson I, Waters K. Physiotherapists working with stroke patients: a national survey. Physiotherapy 2000;86(2):11 
G Mode

GAIPOS-3726; No. of Pages 5

A. Kerr et al./ Gait \& Posture $x x x$ (2012) $x x x-x x x$

5

[7] de Bon EW, Allium JH, Carpenter MG, Salic C, Bloem BR, Conzelmann M, et al. Quantitative assessment of the stops walking while talking test in the elderly. Archives Of Physical Medicine And Rehabilitation 2003;84(6):838-42.

[8] Dion L, Malouin F, McFadyen BJ, Richards CL. Assessing mobility and locomotor coordination after stroke with the rise-to-walk task. Neurorehabilitation and Neural Repair 2003;17:83-92.

[9] Elble R. Gait and dementia: moving beyond the notion of gait apraxia. Journal of Neural Transmission 2007;114(10):5.

[10] Ferris DP, Gordon KE, Sawicki GS, Peethambaran A. An improved powered ankle-foot orthosis using proportional myoelectric control. Gait and Posture 2006;23(4):425-8.

[11] Frykberg GE, Aberg AC, Halvorsen K, Borg J, Hirschfeld H. Temporal coordinatimon of the sit-to-walk task in subjects with stroke and in controls. Archives Of Physical Medicine And Rehabilitation 2009;90(6):1009-17.

[12] Giladi N, Shabtai H, Simon ES, Bran S, TaI J, Korczyn AD. Construction of freezing of gait questionnaire for patients with Parkinsonism. Parkinsonism and Related Disorders 2000;6(3):165-70.

[13] Huang Y-C, Wei S-H, Hsing-Kuo Wang H-K, Lieu F-K. Ultrasonographic guided botulinum toxin type a for plantar fasciitis: an outcome-based investigation for treating pain and gait changes. Journal of Rehabilitation Medicine 2010;42:4.

[14] Hyndman D, Ashburn A. "Stops walking when talking" as a predictor of falls in people with stroke living in the community. Journal of Neurology Neurosurgary and Psychiatry 2004;75(7):994-7.

[15] Kerr A, Rafferty D, Kerr KM, Durward B. Timing phases of the sit-to-walk movement: validity of a clinical test. Gait and Posture 2007;26(1):11-6.

[16] Krasovsky T, Levin MF. Review: toward a better understanding of coordination in healthy and poststroke gait. Neurorehabilitation and Neural Repair 2010; 24(3): 11 .

[17] Krebs HI, Volpe BT, Ferraro M, Fasoli S, Palazzolo J, Rohrer B, et al. Robot-aided neurorehabilitation:from evidence-based to science-based rehabilitation. Topics in Stroke Rehabilitation 2002;8(4):16.

[18] Magnon A, McFadyen BJ, St-Vincent G. Modification of the sit-to-stand task with the addition of gait initiation". Gait and Posture 1996;4(3):232-41.

[19] Malouin F, McFadyen B, Dion L, Richards CL. A fluidity scale for evaluating the motor strategy of the rise-to-walk task after stroke. Clinical Rehabilitation 2003;17(6):674-84.

[20] Morris DM, Uswatte G, Crago JE, Cook EW, Tub E. The reliability of the Wolf Motor Function Test for assessing upper extremity function after stroke. Archives of Physical Medicine and Rehabilitation 2001;82:5.
[21] Pomeroy VM, Pramanik A, Sykes L, Richards J, Hill E. Agreement between physiotherapists on quality of movement rated via videotape. Clinical Rebabilitation 2003;17(3):264-72.

[22] Rahman S, Griffin HJ, Quinn NP, Jahanshahi M. The factors that induce or overcome freezing of gait in Parkinson's disease. Behavioural Neurology 2008;19(3):9.

[23] Rains S, Meadows L, Lynch-Ellerington M. Bobath concept: theory and clinical practice in neurological rehabilitation. New Jersey: John Wiley \& Sons; 2009.

[24] Riley PO, Schenkman ML, Mann RW, Hodge WA. Mechanics of a constrained chair-rise. Journal of Biomechanics 1991;24(1):77-85.

[25] Rohrer B, Fasoli S, Krebs HI, Hughes R, Volpe B, Frontera WR, et al. Movement smoothness changes during stroke recovery. Journal of Neuroscience 2002; 22:8297-304.

[26] Schmidhauser J, Caflisch J, Rousson V, Bucher HU, Latal B. Impaired motor performance and movement quality in very-low-birthweight children at 6 years of age. Developmental Medicine and Child Neurology 2006;48(9):4.

[27] Schneiberg S, McKinley P, Gisel E, Sveistrup H, Levin MF. Reliability of kinematic measures of functional reaching in children with cerebral palsy. Developmental Medicine and Child Neurology 2010;52:6.

[28] Selman TJ, Morris RK, Zamora J, Khan KS. The quality of reporting of primary test accuracy studies in obstetrics and gynaecology: application of the STARD criteria. BMC Women Health 2011;11:8.

[29] Skjaerven LH, Kristoffersen K, Gard G. An eye for movement quality: a phenomenological study of movement quality reflecting a group of thysiotherapists' understanding of the phenomenon. Physiotherapy Theory and Practice 2008;24(1):13-27.

[30] Thelen E. Motor development: a new synthesis. American Psychologist 1995; 50(2): 16.

[31] Tyson SF, Connell LA, Burse ME, Lennon S. What is Bobath? A survey of UK stroke physiotherapists' perceptions of the content of the Bobath concept to treat postural control and mobility problems after stroke. Disability and Rehabilitation 2009;31(6):9.

[32] Zamparo P, Antonutto G, Capelli C, Girardis M, Sepulcri L, di Prampero PE. Effects of elastic recoil on maximal explosive power of the lower limbs. European Journal of Applied Physiology and Occupational Physiology 1997; 75(4):289-97.

[33] Zamparo P, Francescato MP, De Luca G, Lovati L, di Prampera PE. The energy cost of level walking in patients with hemiplegia. Scandinavian Journal of Medicine \& Science in Sports 1995;5(6):348-52.

Please cite this article in press as: Kerr A, et al. Measuring movement fluency during the sit-to-walk task. Gait Posture (2012), http://

dx.doi.org/10.1016/j.gaitpost.2012.09.026 